\title{
Measurement of insulating and dielectric properties of acrylic elastomer membranes at high electric fields
}

\author{
L. Di Lillo, ${ }^{1, a)}$ A. Schmidt, ${ }^{2,3, b)}$ D. A. Carnelli, ${ }^{4}$ P. Ermanni, ${ }^{1}$ G. Kovacs, ${ }^{3}$ E. Mazza, ${ }^{2,3}$ \\ and A. Bergamini ${ }^{3}$ \\ ${ }^{1}$ ETH Zurich, Centre of Structure Technologies, Leonhardstrasse 27, 8092 Zurich, Switzerland \\ ${ }^{2}$ ETH Zurich, Institute for Mechanical Systems, Tannenstrasse 3, 8092 Zurich, Switzerland \\ ${ }^{3}$ Empa, Swiss Federal Laboratories for Materials Testing and Research, 8600 Dubendorf, Switzerland \\ ${ }^{4}$ ETH Zurich, Integrated Systems Laboratory, Gloriastrasse 35, 8092 Zurich, Switzerland
}

(Received 5 July 2011; accepted 28 November 2011; published online 19 January 2012)

\begin{abstract}
This work reports on the investigation of VHB 4910 acrylic elastomer insulating and dielectric properties. This material is widely exploited for the realization of actuators with large deformations, dielectric elastomer actuators (DEA), and belongs to the group of so-called electroactive polymers (EAP). Extensive investigations concerning its mechanical properties are available in literature while its electric behavior at working conditions has not received the same level of attention. In this work, the relative permittivity and the volume resistivity have been measured on VHB 4910 membranes under different fixed stretch conditions $\left(\lambda_{1}, \lambda_{2}=3,3.6,4,5\right)$ using circular gold electrodes sputtered onto both sides of the specimens. The measured values of relative permittivity are in fairly good agreement with the results previously published by other groups. The volume resistivity, at field values close to the operational ones, has shown a fielddependent behavior revealing dissipative properties that should be considered in real applications. Further, measurements on circular actuators verify these findings. Consequences for modeling of VHB 4910 are drawn and new material model parameters proposed to account for the value of relative permittivity at high electric fields. (C) 2012 American Institute of Physics. [doi:10.1063/1.3676201]
\end{abstract}

\section{INTRODUCTION}

A number of adaptive structure applications, such as dielectric elastomer actuators (DEA) and electro-bonded laminates $(\mathrm{EBL}){ }^{1}$, rely on the generation of intense electric fields, in excess of $70 \mathrm{~V} \mu \mathrm{m}^{-1}$. These fields across the thickness of a thin polymer dielectric layer are used to generate a Maxwell stress,

$$
\underline{\sigma}=-\frac{1}{2} \varepsilon_{0} \varepsilon_{r} E^{2} \times\left[\begin{array}{ccc}
1 & 0 & 0 \\
0 & 1 & 0 \\
0 & 0 & -1
\end{array}\right],
$$

where $E$ is the applied electric field, $\varepsilon_{0}$ is the permittivity of vacuum, and $\varepsilon_{\mathrm{r}}$ is the relative permittivity of the material. The Maxwell stress is limited by the maximum electric field the dielectric can withstand without failure, namely the dielectric strength. Figure 1 shows the effect of voltage application to a DEA. The dielectric is highly compliant and deforms in consequence of a charge displacement caused by an external applied electric field. ${ }^{3}$

Thereby, a certain amount of the applied external electric energy is transformed into mechanical energy. The actuation behavior of DEAs is depending on both electric and mechanical properties of the constitutive dielectric layer.

The mechanical characterization of DEA materials, like the $3 \mathrm{M}$ VHB $49 \mathrm{XX},{ }^{4}$ relies on mechanical testing procedures

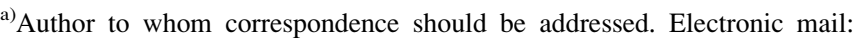
dilillo@mavt.ethz.ch.

b) L. Di Lillo and A. Schmidt contributed equally to the scientific results and to the redaction of this article.
}

(uni- and bi-axial tensile tests, pure shear tests, membrane inflation tests, relaxation and creep tests) and corresponding model equations of their nonlinear behavior. ${ }^{5,6} \mathrm{~A}$ reliable electro-mechanical model of DEA requires the knowledge of both the electrical and the mechanical properties of its dielectric layers.

Permittivity measurements on VHB 4910 have been the purpose of the work of several groups. ${ }^{7-11}$ The typical method used to carry out these measurements relies on the analysis of the capacitive component in an Inductive (L), Capacitive (C), Resistive (R) -LCR- circuit by means of an impedance analyzer (LCR meter), generally operated at low voltage-usually in the range $0-5 \mathrm{~V}$-and frequencies between $100 \mathrm{~Hz}$ and $10^{6} \mathrm{~Hz}$.

There are several aspects of this method that might greatly influence the determination of the dielectric properties of the material under test:

- The relative permittivity of polymers might depend upon the testing frequency, increasing toward lower frequencies. ${ }^{10}$

- The relative permittivity might depend on the electric field intensity $^{11}$ and on the pre-stretch level of the membrane. ${ }^{9,11}$

- The dissipative behavior of polymers might be field dependent. $^{12}$

- The contact conditions between the electrodes and the sample may cause artifacts in the measurement.

As for the last point, which concerns the sample preparation technique rather than the measurement method, the influence on the value of the measured permittivity of air 


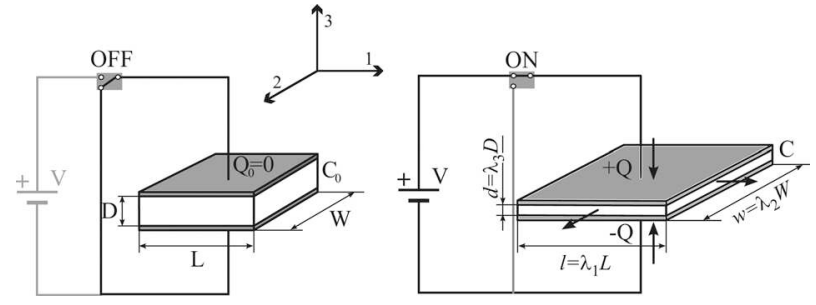

FIG. 1. Working principle of a DEA, adapted from Löwe et al., ${ }^{2}$ displayed in two operating phases: without an external voltage source (left) and with a voltage source (right). The arrows appearing in the right picture stand for the direction of Maxwell stress arising as a consequence of the applied electric field.

trapped between the electrodes and the elastomer will be analyzed.

The frequency of operation and the field intensity at which DEAs and EBLs are activated strongly differ from the conditions under which their dielectric and insulating properties are typically measured. It is possible that at operational conditions, the electrical behavior of DEA and EBL materials differ from the behavior expected following the results achieved through conventional measurement methods. This question motivated the work described in the present paper.

For this purpose we use a novel measurement setup and a signal processing scheme ${ }^{13}$ to determine the relative permittivity and volume resistivity of VHB 4910 acrylic elastomer previously pre-stretched. The novelty factor introduced concerns the conditions of high-field and quasi-static regime at which these properties are measured. This allowed for a detailed investigation of the material behavior close to its potential working conditions.

\section{METHODS}

Measurements were performed at room temperature on several 3M VHB 4910 acrylic elastomer samples prestretched to an equal in-plane stretch at ratios of about $\lambda_{1}=\lambda_{2}=\lambda=3,3.6,4,5$; corresponding to thicknesses of $105 \pm 1 \mu \mathrm{m}, 76 \pm 1 \mu, 62 \pm 1 \mu \mathrm{m}$ and $39 \pm 1 \mu \mathrm{m}$, respectively, and then attached to circular polytetrafluoroethylene (PTFE) frames. These thicknesses were measured in the prestretched state using a precision thickness gauge (MT-30 Heidenhain). The set-up consists of a circular caliper of 6 $\mathrm{mm}$ diameter that is pressed on the samples with a force of $2.2 \mathrm{~g}$. The position of the caliper is detected with a capacitive sensor having a resolution of $\pm 0.5 \mu \mathrm{m}$.

VHB 4910 is commercially available in the form of a membrane with $1 \mathrm{~mm}$ initial thickness, $230 \mathrm{~mm}$ width, and arbitrary length. The chemical composition is not declared by the manufacturer. On each VHB membrane three pairs of $20 \mathrm{~nm}$ thick (estimation based on the sputtering parameters) gold electrodes and $20 \mathrm{~mm}$ diameter, named $A, B, C$ throughout the text, were applied by a sputtering process in a controlled environment to avoid any defects/structural changes of the thin layers, Fig. 2. The sputtering process was carried out using a Baltec 005 sputtering device at a sputtering current of $15 \mathrm{~mA}$ for $300 \mathrm{~s}$, a pressure of $40 \mathrm{mbar}$ in an argon atmosphere, and a target distance of approximately $50 \mathrm{~mm}$.

In order to suppress the deformation of the dielectric and the consequent failure of the sputtered gold electrodes,

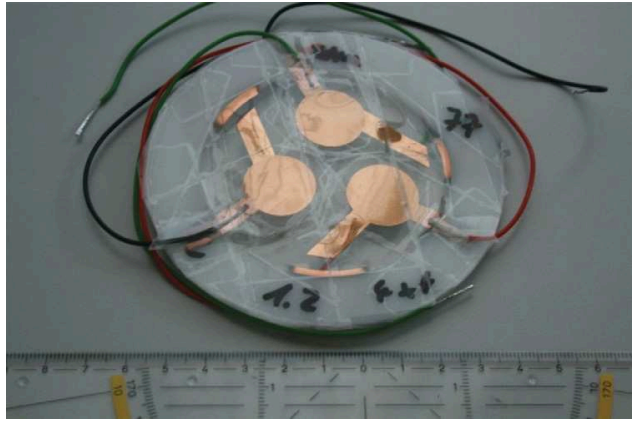

FIG. 2. (Color online) A $4 \times 4$ pre-stretched VHB 4910 membrane. The three active areas had $20 \mathrm{~nm}$ thick gold sputtered electrodes and, on top of the whole surface, the adhesive tape was used as a stiffening means. The VHB adhesive membrane is mounted on PTFE circular frames with a 70 mm inner diameter.

caused by the application of the voltage, the samples were stiffened in the in-plane directions on both sides with common adhesive tape.

Figure 3 shows the set-up used for measuring the current $I(t)$. The voltage time history is generated by programming a suitable signal into the signal generator (Agilent $33120 \mathrm{~A}$ Arbitrary Waveform generator). The signal is amplified by a high voltage amplifier (Trek 20/20C) and applied to the samples. The samples respond by becoming polarized by various mechanisms (e.g. ionic, orientational) and reducing the resulting electric field across them. The current, carrying information about the degree of charge displacement and conduction mechanisms within the samples, is converted to a voltage signal by a trans-impedance amplifier built in the laboratory and recorded by an oscilloscope (Tektronix TDS5034B). The noise introduced by the high voltage amplifier is reduced by combining a proper digital filter with a physical shielding of the system.

Measurements were performed to different target voltages that decreased with increasing value of pre-stretch $\lambda$. The maximum target voltage $V_{\max }$ for $\lambda=3$ was $5000 \mathrm{~V}$, for $\lambda=3.6 V_{\text {max }}$ was $4000 \mathrm{~V}$, and for $\lambda=5 V_{\text {max }}$ was $3000 \mathrm{~V}$. Each plot shown in this article is the result of 5 consecutive measurements carried out on the same area, unless stated otherwise.

The relative permittivity $\varepsilon_{r}$ of the dielectric elastomer is directly proportional to the charging current $I_{c}$, as shown in Eq. (2),

$$
\varepsilon_{r}=\frac{I_{c} d}{\varepsilon_{0} A \dot{V}} .
$$

In Eq. (2), $d$ is the thickness of the elastomer, $\varepsilon_{0}$ is the vacuum permittivity, $A$ is the area of the sample, and $\frac{d V}{d t}=\dot{V}$ is the slope of the applied voltage ramp. $\dot{V}$ was kept constant at 200 $\mathrm{V} \mathrm{s}^{-1}$ for all measurements reported in this work. This parameter and the maximum value of the voltage signal applied, $V_{\text {max }}$, define, by a Laplace transformation, the frequency $\frac{\dot{V}}{V_{\max }}(\mathrm{Hz})$, at which most of the signal energy is centered.

Circular actuators with pre-stretch of $\lambda_{1}=\lambda_{2}=3,4,5$ have been prepared by applying a thin layer of carbon powder to a circular region of diameter $10 \mathrm{~mm}$ centered onto the pre-stretched membranes (outer diameter $70 \mathrm{~mm}$ ). 


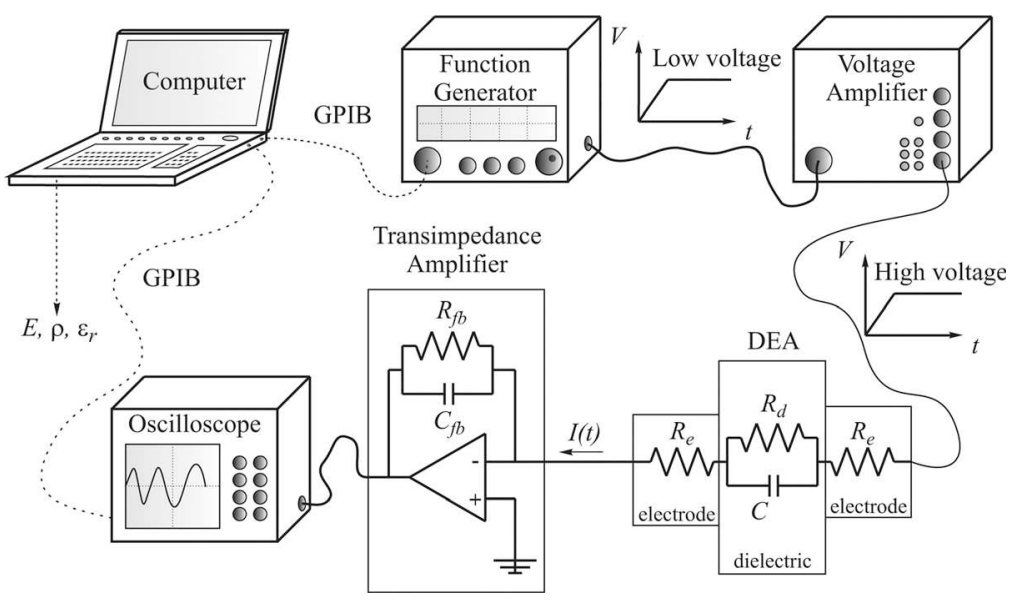

FIG. 3. High voltage measurement setup, adapted from Di Lillo et al. (Ref. 13), used for determining the insulating and dielectric properties of VHB 4910 acrylic elastomer membranes, being the constitutive elements of DEA. The picture outlines the main circuitry elements used for modeling the $I(t)$ behavior of the membranes.

\section{RESULTS}

\section{A. Volume resistivity}

Fig. 4 qualitatively depicts the charging current (Fig. 4(b)) for a capacitor on which a linear voltage ramp (Fig. 4(a)) is imposed, for conditions of ideal (flat curve in Fig. 4(b)) and non-ideal insulation (ascending curve during the voltage ramp which becomes non-zero at constant voltage in Fig. 4(b)).

Representative current responses of a $5 \times 5$ stretched VHB 4910 film exposed to two different voltage ramps, $1000 \mathrm{~V}$ and $3000 \mathrm{~V}$-corresponding to fields of $25 \mathrm{~V} \mathrm{\mu \textrm {m } ^ { - 1 }}$ and $75 \mathrm{~V} \mu \mathrm{m}^{-1}$, respectively-are illustrated in Fig. 5. The signals shown in Fig. 5(a) appear nearly perfectly superimposed, indicating the absence of charge build-up in the dielectric elastomer and underlying the lack of any appreciable remaining polarization. Indeed, this behavior fits fairly well the one of an ideal capacitor having only a slight positive slope, which reveals its ohmic nature.

As for the high field measurements, show in Fig. 5(b), the current remarkably deviates from the behavior of the two situations presented in Fig. 4(b). Further, it is noticeable how the first measurement (highlighted as "1st loop" in Fig. 5(b)) differs from the subsequent ones. This remarkable difference can be due to a slight reduction of the polymer thickness, which turns into having a higher capacitance and, thereby, a higher value of the current in the successive loops. This behavior has been recorded on all the $5 \times 5$ samples tested and it became evident from applied fields higher than $60 \mathrm{~V} \mu \mathrm{m}^{-1}$. A similar behavior has not been observed on the samples with different stretches-i.e., $3 \times 3$ and $3.6 \times 3.6$-for which we did not
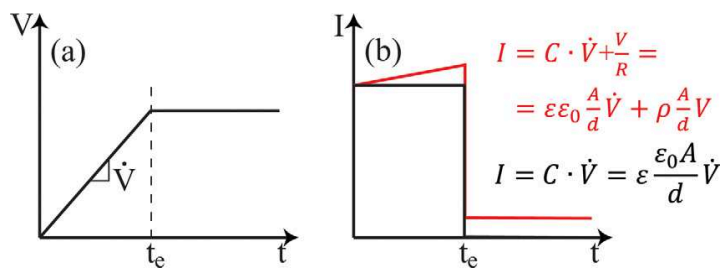

FIG. 4. (Color online) $I(t)$ expected (flat curve in (b)) response of an ideal capacitor with an area $A$, vacuum permittivity $\varepsilon_{0}$, relative permittivity $\varepsilon_{\mathrm{r}}$, and thickness $d$ exposed to a $V(t)$ voltage signal (a) having a slope $\dot{V}$. The $I(t)$ real response (ascending curve during the voltage ramp which becomes nonzero at constant voltage in (b)) of a capacitor accounts for the non-infinite insulation resistance $R$ of the material. reach the same field intensity as for the $5 \times 5$ samples. We did not apply higher fields on samples with lower stretch levels because it would have been highly likely to observe a breakdown event on such samples. Indeed, the probability to observe a breakdown event is high as the dielectric strength is not only dependent on the electric properties of the polymer but also on the presence of flaws. ${ }^{14}$ Thereby, the thicker the sample the lower the dielectric strength. ${ }^{9}$

The current appearing in the constant-voltage regime is the clear signature of the conductive behavior of the dielectric elastomers. It is, indeed, the expression of the finite VHB 4910 volume resistivity. Figure 5 shows that the current has a field-dependent behavior.
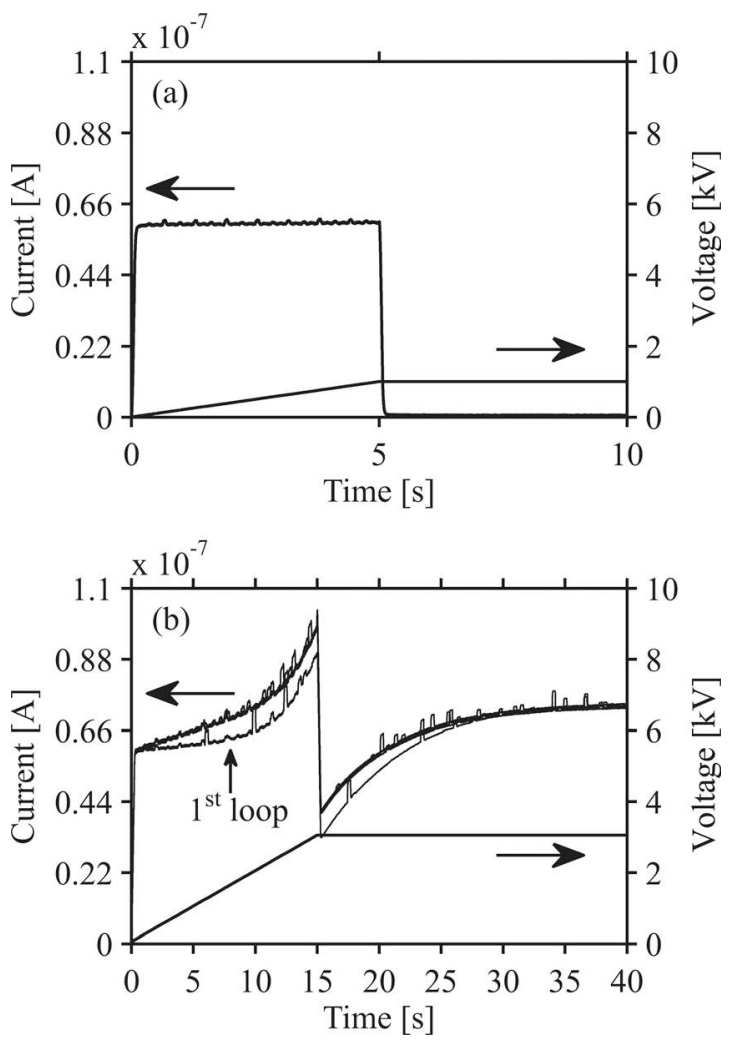

FIG. 5. (a) The $I(t)$ response of a $5 \times 5$ pre-stretched VHB 4910 membrane (sample $C$ ) exposed to a $V(t)$ voltage rising with a slope of $200 \mathrm{~V} \mathrm{~s}^{-1}$ and a target voltage of $1000 \mathrm{~V}$ applied through $20 \mathrm{~mm}$ diameter gold sputtered electrodes. (b) The $I(t)$ response of the same $5 \times 5$ membrane exposed to a $V(t)$ signal having the same slope, but a target voltage of $3000 \mathrm{~V}$. 


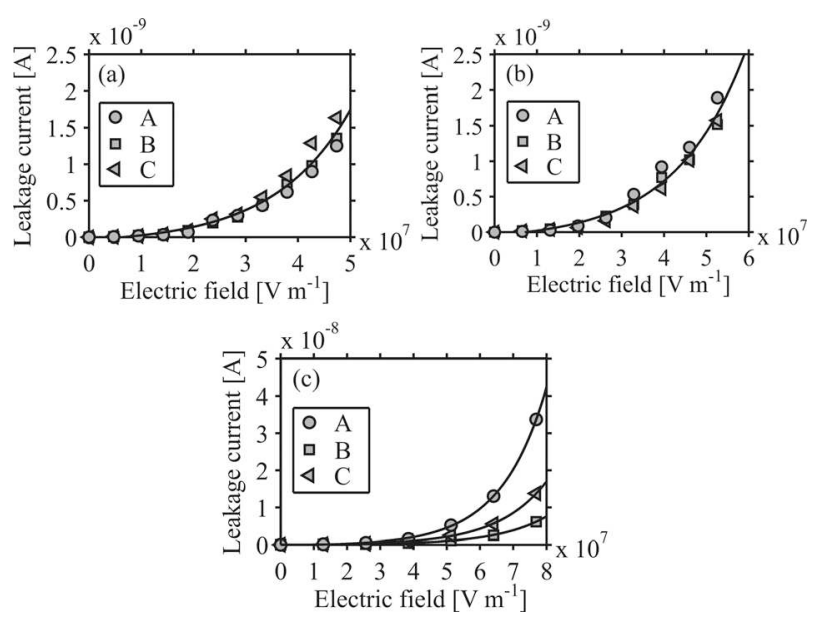

FIG. 6. Measured (symbols) leakage current of three different samples with pre-stretch $3 \times 3$ (a), $3.6 \times 3.6$ (b), $5 \times 5$ (c), and the corresponding exponential fit model (line).

Thereby, for computing the value of the relative permittivity of the samples tested at high fields, the model presented by Di Lillo et al. ${ }^{13}$ (which remains valid and very accurate for polymer materials having an ohmic behavior) has to be extended and should account for the leakage current to be subtracted during the voltage ramp. To accomplish that, we extracted and plotted a field-dependent leakage current for each sample capturing the $I(t)$ trends at different constant voltages (500-5000 V), Fig. 6.

Figure 6 shows the exponential behavior of the leakage current for the whole set of samples tested and the fairly large spreading between the values recorded for the $5 \times 5$ pre-stretched samples. According to Gisby et al., ${ }^{12}$ this spreading can be due to impurities or voids within the membrane or to local changes in its thickness. Consequently, the volume resistivity of the material, shown in Fig. 7, shows a strong dependence on the applied $E$ (to be noticed in the logarithmic scale for the vertical axis).

\section{B. Relative permittivity}

Figure 8 summarizes the relative permittivities $\varepsilon_{r}$ as determined from current responses for all the VHB 4910 dielectric elastomers examined.

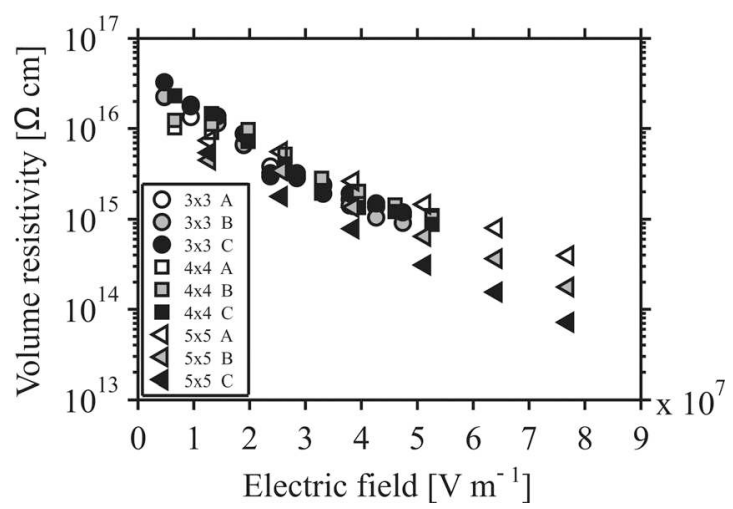

FIG. 7. Volume resistivity trend $\rho(\Omega \mathrm{cm})$ for the whole set of samples tested. Each point corresponds to the mean value of 5 consecutive measurements.

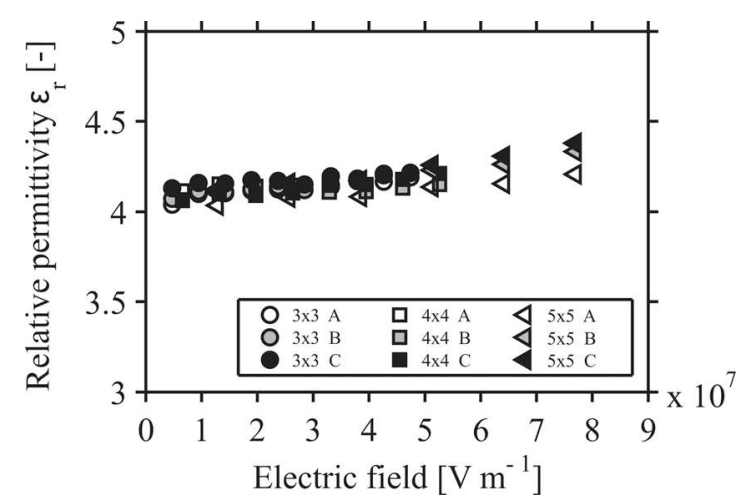

FIG. 8. Trend of the dielectric response, relative permittivity $\varepsilon_{r}$, for the whole set of samples tested. Each point corresponds to the mean value of 5 consecutive measurements.

Each point in Fig. 8 represents the average value of $\varepsilon_{r}$ with a standard deviation smaller than $1 \%$. All tested samples, regardless of their pre-stretched states, exhibit a value of $\varepsilon_{r}$ slightly increasing with the field intensity up to the $E_{\max }$ applied. The average value of $\varepsilon_{r}$ extracted for all of them is between 4.05 and 4.2 .

The measurements performed have not evidenced any remanent polarization build-up at elevated electric fields. This is indicative of the non-polar nature of the VHB 4910 samples tested.

\section{Influence of the electrode-sample arrangement on the measured $\varepsilon_{\mathrm{r}}$}

To have a qualitative and quantitative picture of the influence of the electrode-sample arrangement on the measurements, we tested a $4 \times 4$ stretched VHB 4910 sample, shown in Fig. 9(a), using the same brass electrodes used for the LCR measurements by Wissler and Mazza. ${ }^{7}$ Figure 9(b) shows the electric behavior of a $3.6 \times 3.6$ stretched VHB 4910 sample with gold electrodes sputtered onto both sides. The $I(t)$ plots of Figs. 9(a) and 9(b) are composed of 5 successive measurements acquired with the samples being exposed to a voltage signal ramping at a rate of $200 \mathrm{~V} \mathrm{~s}^{-1}$ up to a $V_{\max }$ of $2500 \mathrm{~V}$.

Figure 9(a) shows two distinct sectors: sector 1, which provides a value of relative permittivity of $\varepsilon_{r}=2.68$; and sector 2 , which instead yields a value of relative permittivity of $\varepsilon_{r}=4.11$. The abrupt increase of the current at $5 \mathrm{~s}$, corresponding to an applied voltage of $1000 \mathrm{~V}$, is thought to be a consequence of the air ionization, happening at fields of about 3-4.5 $\mathrm{V} \mu \mathrm{m}^{-1}$, see Lide. ${ }^{15}$ The ionization of air causes a release of free carriers, which affects the density of bound charges at the membrane surface. To keep up a constant voltage, more charges should be pumped from one electrode to the other causing, thereby, an abrupt increase in the current signal. The influence of the same air inclusion on the calculated permittivity will be higher for smaller thicknesses (i.e., higher pre-stretch) of the sample. This explains the fact that measurements with stiff electrodes lead to permittivity values strongly decreasing with in-plane stretch. ${ }^{7}$

The different leakage current between the two configurations in Fig. 9 stems from geometrical considerations-the 

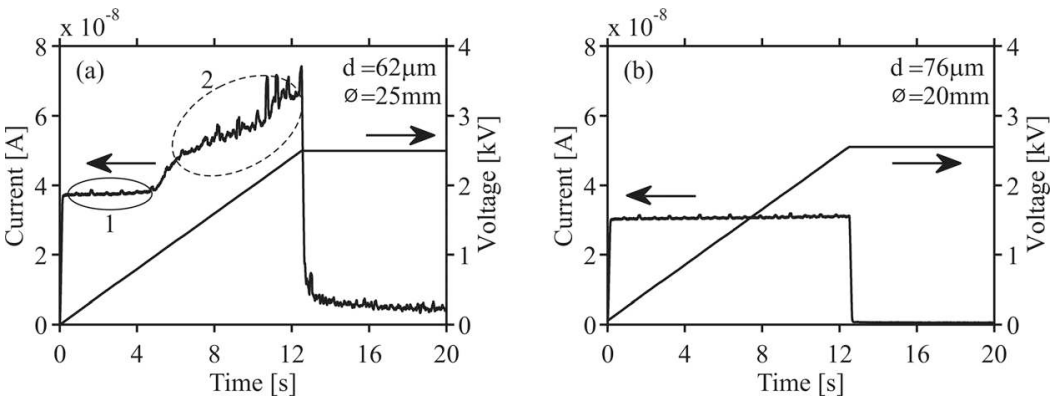

FIG. 9. (a) The $I(t)$ response of a $4 \times 4$ pre-stretched VHB 4910 membrane exposed to a $V(t)$ voltage rising with a slope of $200 \mathrm{~V} \mathrm{~s}^{-1}$ and a target voltage of $2500 \mathrm{~V}$ applied through the same system with brass electrodes presented by Wissler and Mazza ${ }^{7}$ having a diameter $\varnothing$ of $25 \mathrm{~mm}$. Two areas -1 and 2-have been highlighted, representing the $I(t)$ behavior before and after a presumable ionization process of trapped air, which gives rise to a significant change in the value of the measured relative permittivity. (b) The $I(t)$ behavior of a $3.6 \times 3.6$ pre-stretched VHB 4910 membrane exposed to a $V(t)$ having the same features detailed above through gold sputtered electrodes having a diameter $\varnothing$ of $20 \mathrm{~mm}$. slight differences in the electric field intensity due to diverse pre-stretch levels and the diverse electrode diameters-and material considerations, the electrodes having an influence on the conductive properties of the material under test. ${ }^{16}$

\section{Electromechanical behavior of circular actuators}

A voltage history with an initial ramp of $200 \mathrm{~V} \mathrm{~s}^{-1}$ up to final values of $2000 \mathrm{~V}$ to $3500 \mathrm{~V}$ followed by a period with a constant voltage for $100 \mathrm{~s}$ was applied to the samples while the current was recorded. Simultaneously, the radial strain was measured using a camera and the reported results, shown in Fig. 10, are in excellent agreement with the data presented by Wissler and Mazza. ${ }^{6}$

Figure 11 shows the measured radial strain, voltage, and current of a circular actuator with pre-stretch $5 \times 5$ loaded with a voltage of $1000 \mathrm{~V}$ (Fig. 11(a)) and $3000 \mathrm{~V}$ (Fig. 11(b)). Further, the capacitance calculated assuming a constant permittivity of 4.1 is reported. As shown in Fig. 11, within the initial period an increasing voltage causes a nonlinearly increasing strain. When the voltage is held constant, the strain further increases due to viscoelasticity of the material and the increase of the electric field caused by the thinning of the material. The measured current rises sharply at the beginning of the experiment and further increases during the voltage ramp. The latter effect is a result of the increasing capacitance of the actuator caused by the deformation (thickness reduction and in-plane expansion). Further, the exponential dependence of leakage current on
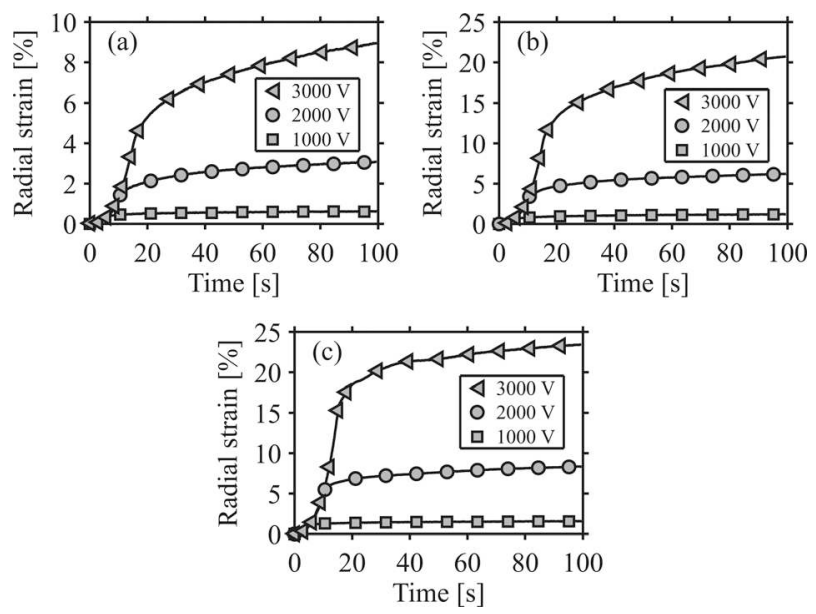

FIG. 10. Measured radial strain on circular actuators with $\lambda_{1}=\lambda_{2}=3$ (a), 4 (b) and 5 (c). the electric field, shown in Fig. 6, contributes to the increasing current especially for high electric fields (Fig. 11(b)). This is emphasized by the considerable amount of current that is observed in the phase of a constant voltage where the capacitance changes only a little.

\section{DISCUSSION}

Gisby et al $^{12}$ reported on the relationship between electric field and leakage current for analyzing and possibly predicting/preventing a breakdown event in VHB 4905 membranes stretched equibiaxially to 16 times their area. The leakage currents reported are qualitatively comparable to the ones presented in this paper for samples with similar thickness, i.e., Fig. 6(c). Indeed, an exponential behavior and a relatively large variation between the curves were observed
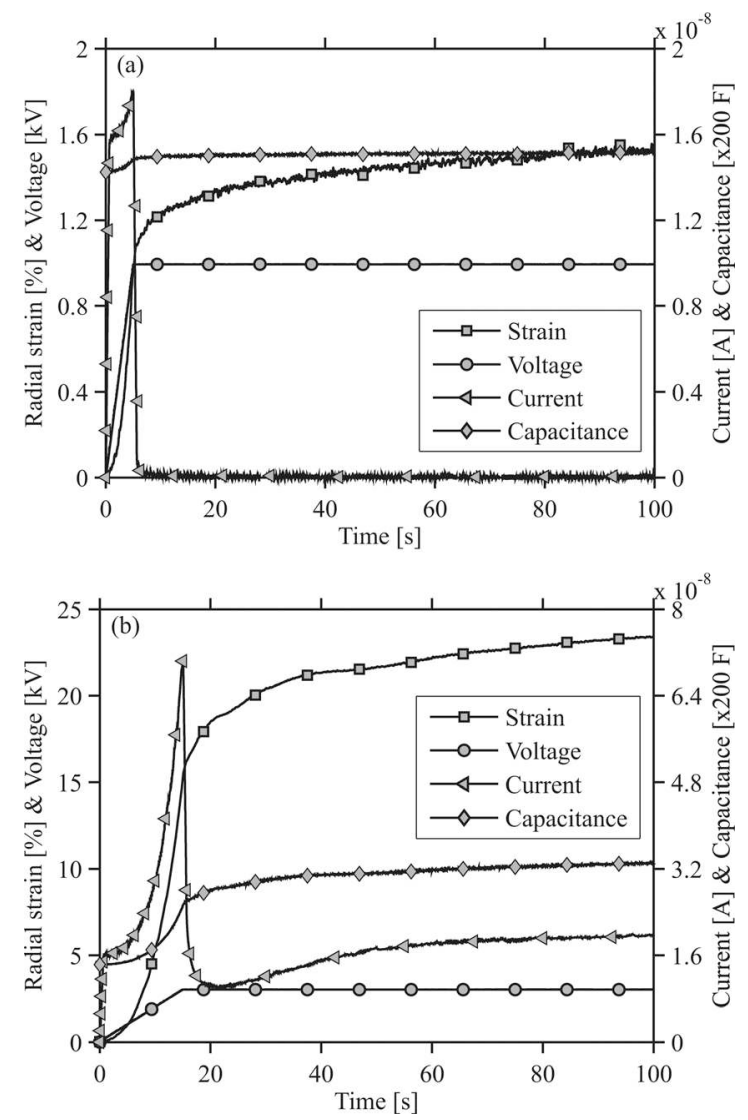

FIG. 11. Measured current, strain, and voltage and calculated capacitance of a circular actuator with pre-stretch $5 \times 5$ subjected to a maximum voltage of $1000 \mathrm{~V}$ (a) and $3000 \mathrm{~V}$ (b). 
by Gisby et al. ${ }^{12}$ and described as being the effect of voids or thickness variations throughout the sample or impurities. Actually, the presence of impurities creates localized states and traps for the electrons in the energy bandgap of the electronic band structure of the dielectric elastomer. This circumstance strongly affects the value of the dielectric strength, as it increases the probability to have breakdown phenomenon at a field intensity lower than expected. The value of leakage current/volume resistivity too is affected because it creates further conductive channels. Quantitatively, the values of current recorded by Gisby et al. ${ }^{12}$ are much higher than the ones reported in this work, mainly because of the higher fields imposed on the samples. With the electric field increasing, more electrons will gain enough energy to jump from the valence band to the unoccupied states of the conduction band having, then, the capability to contribute to the density of current flowing through the sample. ${ }^{17}$ The dielectric strength and the volume resistivity are intimately connected and both provide information about the strength of the electron-cloud-nucleus bond. ${ }^{17}$ Apart from the bandgap related considerations, the conductive properties of polymers are affected by other mechanisms, which are peculiar of their particular chemical and physical structure. The study of these mechanisms, like space charge formation ${ }^{18-20}$ and the consequences of impurities ionization, structural discontinuities, and crystalline/amorphous interfaces, ${ }^{14}$ were beyond the scope of this work. For thicker samples and lower field values, representative of actuator working conditions, the variability of leakage current is much lower, as shown in Figs. 6(a) and 6(b). Under these conditions, the predictability of volume resistivity is much better; see Fig. 7 (circles and squares).

A further observation concerns the time-dependent behavior of the leakage current in Fig. 5(b). Indeed, the current clearly drops as soon as the applied voltage turns to a constant value but increases afterward. Significant deformations can be excluded in this experiment. The time dependence, which points out the non-Ohmic nature of the samples at high fields, may thus be due to the onset of localized thermal effects following the application of the high field intensity that the dielectric elastomer has to withstand. At this stage, this hypothesis has not been confirmed and it will be the subject of further investigations.

As for $\varepsilon_{r}$, McKay et al. ${ }^{11}$ used different techniques, LCR and blocked force, and different electrodes to investigate the relative permittivity of both unstretched and stretched VHB 4905. Particularly, they sputtered gold electrodes to ensure the absence of air voids otherwise supposed to be present if using paint brushed carbon grease electrodes. Indeed, they observed a larger reduction of $\varepsilon_{r}$ with area stretch for samples having carbon grease electrodes as compared with those having gold sputtered electrodes. The modest decrease of $\varepsilon_{r}$ with stretch they measured with gold sputtered electrodes was similar to that presented by Kofod et al. ${ }^{9}$, ranging from 4.49 (unstretched) to 4.20 ( $4 \times 4$ stretch level). The values of $\varepsilon_{r}$ reported in our work are within a similar range for all the stretches we tested (there is no confirmation of stretch dependency) but at slightly lower values, i.e., 4.05-4.2.
Through the measured values of volume resistivity $\rho(\Omega \mathrm{cm})$ and relative permittivity $\varepsilon_{r}$, the stored energy density (Fig. 12(a)) and dissipated power density (Fig. 12(b)) of the tested samples have been calculated. These two parameters are of valuable importance for the correct design of DEA actuators and energy harvesting devices.

The calculated stored energy density increases quadratically with the field, as shown in Fig. 12(a), as can be expected for a material with field-independent relative permittivity (an average relative permittivity $\varepsilon_{r}=4.1$ has been considered for all the samples). This turns into having a maximum energy density that can be stored in a certain actuator, exploiting the VHB 4910 as active medium, depending only on its dielectric strength, which is stretch dependent. ${ }^{9}$

The instantaneous power is the product of $I(t)$ and $V(t)$, and it can be argued that, once the system has been charged up, the charging power should drop to zero assuming then the power consumption being nearly zero. This consideration stems from the assumption that the volume resistivity of the polymer was extremely high, as it is indeed at low field intensities, as seen in Fig. 5(a). However, at high fields, the volume resistivity of the samples tested strongly decreases, resulting in a dissipated power that appears to increase in an exponential manner with respect to the applied field, Fig. 12(b). This behavior could point out the existence of an activation energy, above which some space charges are setting up in the material, leading to a consequent exponential
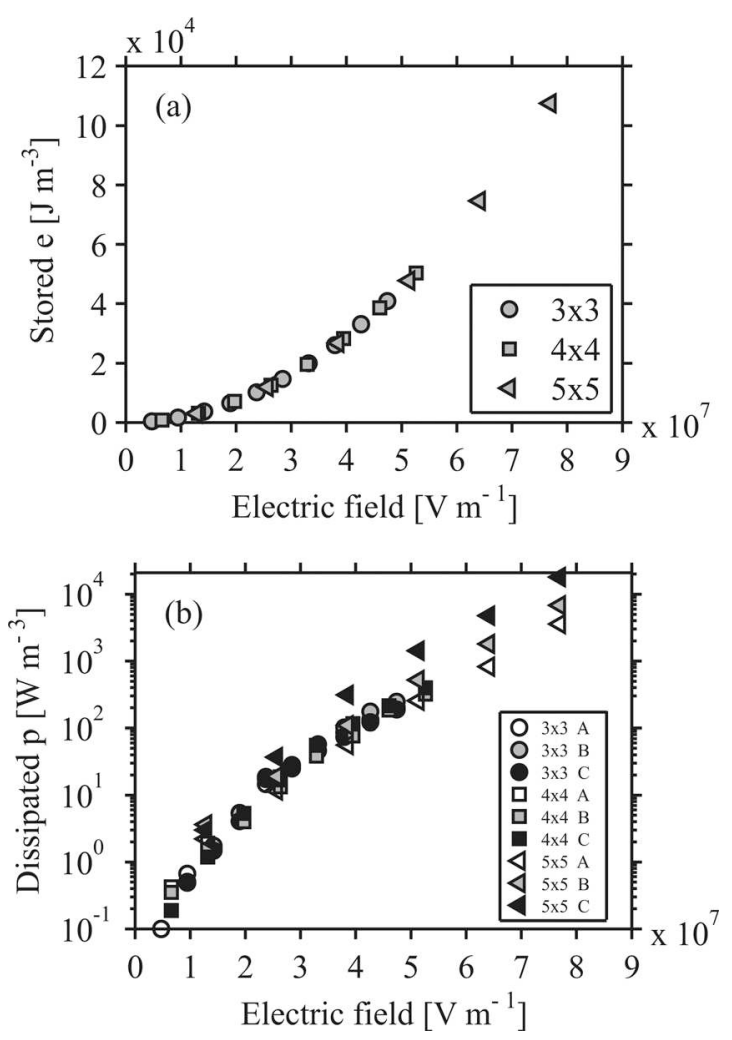

FIG. 12. (a) The stored electric energy density $e$ for the VHB 4910 tested samples as calculated based on an average relative permittivity $\epsilon_{r}=4.1$, as a function of the applied electric field $E$. (b) The dissipated power density $p$ calculated through the applied voltage signal and the measured values of volume resistivity $\rho(\Omega \mathrm{cm})$ are shown in $\log$ scale, as functions of the applied electric field $E$. 
increase of the leakage current. However, an in-depth analysis of such mechanisms requires gaining access to the chemical composition of these acrylic elastomers.

\section{A. Electromechanical modeling of VHB $\mathbf{4 9 1 0}$}

The description of the electromechanical behavior of DEA requires knowledge of the passive mechanical as well as the electric properties of the material. The phenomenological nature of common nonlinear elastic material models involves a general uncertainty when modeling stress states other than those characterized with experiments. In order to increase the reliability of the material model, its parameters should, therefore, be determined in the same or similar stress states for which modeling is intended. For DEA applications of VHB 4910, loading is caused by the electric field and in most cases by mechanical pre-stretching. The experimental characterization of the resulting multiaxial stress states in a purely mechanical experiment is very difficult. Thereby, mechanical material parameters were derived from a combination of passive mechanical and electromechanical experiments. ${ }^{6}$ Specifically, in Wissler and Mazza ${ }^{6}$ the measured radial expansion of pre-stretched circular actuators was compared with predictions of finite-element models. By iteratively adapting the mechanical material parameter, hyperelastic-viscoelastic material models were determined. Corrected values of the mechanical material model parameters were reported, ${ }^{7,21}$ based on a constant permittivity of 3.2 that represents an average value of the strain dependent permittivity. ${ }^{7}$ In Zhao and Suo, ${ }^{22}$ consequences of a deformation-dependent permittivity onto the electromechanical coupling are drawn. It is shown that additionally to the Maxwell stress, an electrostrictive component related to the partial derivative of permittivity with respect to deformation adds to the electromechanical coupling for nonideal dielectrics. In the case of VHB 4910 the apparent dependence of permittivity on deformation ${ }^{7}$ is now shown to be due to a measurement artifact, probably caused by air inclusions between the samples and the rigid brass electrodes. We now consider VHB 4910 to follow the behavior of an ideal dielectric and thus electromechanical coupling can simply be expressed by Eq. (1). A mechanical model based on this assumption and on the measured value of relative permittivity can therefore be determined.

\section{B. Influence of the measured permittivity on the mechanical models}

In Wissler and $\mathrm{Mazza}^{7}$ a quasi-linear viscoelastic material behavior is used. Only the elastic part of the model is affected by the new findings. A dependence of the relative permittivity on the electric field is neglected for the present calculations. Maxwell stress is directly proportional to the relative permittivity and, for a given electric field, does not depend on deformation. The hyperelastic coefficients determined by the fitting process described by Wissler and Mazza, ${ }^{6}$ therefore, scale with the value of relative permittivity. A simple correction of these parameters is thus possible using the new determined value of permittivity of $\varepsilon_{r}=4.1$. The reliability of the material model response for circular
TABLE I. Hyperelastic parameters (instantaneous response) for strain energy formulations according to Arruda and Boyce, ${ }^{23}$ Odgen, ${ }^{24}$ and Yeoh. ${ }^{25}$ Values from Wissler and Mazza ${ }^{6}$ are reported in parentheses.

\begin{tabular}{ll}
\hline \hline Arruda and Boyce \\
\hline$W=A\left(\begin{array}{l}\frac{1}{2}\left(I_{1}-3\right)+\frac{1}{20 N}\left(I_{1}^{2}-9\right)+\frac{11}{1050 N^{2}}\left(I_{1}^{3}-27\right) \\
+\frac{19}{7000 N^{3}}\left(I_{1}^{4}-81\right)+\frac{1}{673750 N^{4}}\left(I_{1}^{5}-243\right)\end{array}\right)$ \\
$A[\mathrm{MPa}]$ & $0.0598(0.0686)$ \\
$N$ & $124.88^{\mathrm{a}}$ \\
Ogden & \\
$W=\sum_{i=1}^{3} \frac{\mu_{1}}{\alpha_{1}}\left(\lambda_{1}^{\alpha_{i}}+\lambda_{2}^{\alpha_{i}}+\lambda_{3}^{\alpha_{i}}-3\right)$ & \\
$\mu_{1}[\mathrm{MPa}]$ & $7.48 \times 10^{-3}\left(8.58 \times 10^{-3}\right)$ \\
$\alpha_{1}$ & $1.293^{\mathrm{a}}$ \\
$\mu 2[\mathrm{MPa}]$ & $0.0735(0.0843)$ \\
$\alpha_{2}$ & $2.325^{\mathrm{a}}$ \\
$\mu_{3}[\mathrm{MPa}]$ & $-0.0203(-0.0233)$ \\
$\alpha_{3}$ & $2.561^{\mathrm{a}}$ \\
Yeoh & \\
$W=C_{1}\left(I_{1}-3\right)+C_{2}\left(I_{1}-3\right)^{2}+C_{3}\left(I_{1}-3\right)^{3}$ \\
$C_{1}[\mathrm{MPa}]$ & $0.0721(0.0827)$ \\
$C_{2}[\mathrm{MPa}]$ & $-6.52 \times 10^{-4}\left(-7.47 \times 10^{-4}\right)$ \\
$C_{3}[\mathrm{MPa}]$ & $5.11 \times 10^{-6}\left(5.86 \times 10^{-6}\right)$ \\
\hline \hline
\end{tabular}

${ }^{\mathrm{a}}$ Values unaffected by corrections.

actuators $^{6,21}$ is unaffected by these corrections. A summary of corresponding hyperelastic parameters for the relevant strain energy formulations ${ }^{7}$ is reported in Table I.

\section{CONCLUSIONS}

The electric behavior of commercially available VHB 4910 acrylic elastomer films has been investigated through accurate charging current measurements, which revealed their dielectric and insulating response, specifically the relative permittivity and the volume resistivity, under quasi-static high field conditions. These properties have been investigated on films at pre-stretch and field levels comparable to the ones found in real applications of this material for DEAs.

The results obtained for the relative permittivity $\varepsilon_{r}$ are in the range of the values published by other groups. ${ }^{8-11,21}$ The previously reported deformation-dependent behavior of the relative permittivity $\varepsilon_{r}$ for VHB 4910 samples was not confirmed. This result is supposed to be a consequence of the fact that, unlike previous measurements where solid electrodes were leaned onto the elastomer surfaces, assuming an intimate contact, in this work the electrodes were applied directly to the material by a sputtering process providing, then, a more accurate and precise picture of the material behavior.

The measurement of the volume resistivity $\rho(\Omega \mathrm{cm})$ of VHB 4910 at high electric fields as a function of the stretch conditions $\lambda$ is considered to be a useful contribution to the understanding of this widely used material. The results reported in Fig. 7 are in good agreement with the volume resistivity values reported by Molberg et al. ${ }^{10}$ at fairly low electric field levels. The values recorded for high electric field intensities show an aspect of the behavior of this material that had not been previously reported based on direct electrical measurements. An indirect evidence of such a dissipative behavior of VHB 4910 samples has been 
brought out by Plante and Dubrowsky ${ }^{26}$ through mechanical measurements.

The evaluation of the stored energy densities $e\left(\mathrm{~J} \mathrm{~m}^{-3}\right)$ and dissipated power densities $p\left(\mathrm{~W} \mathrm{~m}^{-3}\right)$ with respect to the applied field, shown in Figs. 12(a) and 12(b) respectively, points out that, for the operation of DEAs using VHB 4910, the dissipative electrical behavior of the material cannot be neglected when making considerations about the efficiency of these systems. This is expected to have as well a remarkable influence in energy harvesting devices having these materials as constitutive elements. Indeed, the data reported here are useful in determining optimal/suitable modes of operation for DEA and energy harvesting.

We analyzed the consequences for the modeling of VHB 4910 and we corrected the previously proposed mechanical material models to account for the true value of relative permittivity. The electric behavior of circular DEAs during operation has been recorded, confirming the electric energy dissipation at high electric fields.

The experiments we reported in this work indicate that both the relative permittivity $\varepsilon_{r}$ and the volume resistivity $\rho$ $(\Omega \mathrm{cm})$ of VHB 4910 acrylic elastomer membranes can be regarded as not stretch dependent.

Future work should take up the investigation of the dielectric and insulating properties of other candidate materials for DEA applications and compare them to the ones of VHB 4910. The mechanisms leading to the non-Ohmic behavior of VHB will require a more thorough investigation, especially in view of the development of materials with improved insulating properties. Another factor to take into account for future work concerns the implications that the thermal effects may have on the durability of DEAs, especially in the case of devices with low specific surfaces, such as stacked actuators. ${ }^{27}$

\section{ACKNOWLEDGMENTS}

This project was partly funded by the Swiss National Science Foundation, National Research Program 62, Smart Materials under Grant Agreement No. 406240_1261130 and partly by the Swiss National Science Foundation under Grant Agreement No. 200021-107661. The authors wish to acknowledge the financial support they received for this work. Rebekka Ginés from the Structural Engineering Laboratory of Empa and Günter Grossmann from Electronics Laboratory of Empa are acknowledged for their valuable support in sample preparation.

${ }^{1}$ A. Bergamini, R. Christen, B. Maag, and M. Motavalli, Smart Mater. Struct. 15, 678 (2006).

${ }^{2}$ C. Löwe, X. Zhang, and G. Kovacs, Adv. Eng. Mater. 7, 361 (2005).

${ }^{3}$ Z. Suo, Acta Mech. Solida Sinica 23, 549 (2010).

${ }^{4}$ The VHB 49XX membranes used in this paper are registered trademarks of $3 \mathrm{M}^{\mathrm{TM}}$.

${ }^{5}$ A. Schmidt, A. Bergamini, G. Kovacs, and E. Mazza, Exp. Mech., 51 1421 (2011).

${ }^{6}$ M. Wissler and E. Mazza, Sens. Actuators, A 134, 494 (2007).

${ }^{7}$ M. Wissler and E. Mazza, Sens. Actuators, A 138, 384 (2007).

${ }^{8}$ C. Jean-Mistral, A. Sylvestre, S. Basrour, and J. Chaillout, Smart Mater. Struct. 19, 075019 (2010).

${ }^{9}$ G. Kofod, P. Sommer-Larsen, R. Kornbluh, and R. Pelrine, J. Intell. Mater. Syst. Struct. 14, 787 (2003).

${ }^{10}$ M. Molberg, Y. Leterrier, C. Plummer, C. Walder, C. Lowe, D. Opris, F. Nuesch, S. Bauer, and J. Manson, J. Appl. Phys. 106, 054112-054112 (2009).

${ }^{11}$ T. McKay, E. Calius, and I. Anderson, Proc. SPIE 7287, 72870P (2009).

${ }^{12}$ T. Gisby, S. Xie, E. Calius, and I. Anderson, Proc. SPIE 7642, 764213 (2010).

${ }^{13}$ L. Di Lillo, D. A. Carnelli, A. Bergamini, S. Busato, and P. Ermanni, Smart Mater. Struct. 20, 057002 (2011).

${ }^{14} \mathrm{M}$. Chanda and S. Roy, Plastics Technology Handbook (CRC, Boca Raton, Florida, 2006).

${ }^{15}$ D. Lide, Handbook of Chemistry and Physics (CRC, Boca Raton, FL, 1994).

${ }^{16}$ M. Ieda, IEEE Trans. Electr. Insul., 261 (1987).

${ }^{17}$ N. Ashcroft and N. Mermin, Solid Sate Physics (Holt Rinehart and Winston, New York, 1976).

${ }^{18}$ M. Lisowski, R. Kacprzyk, E. Motyl, and P. Gałezki, in Proceedings of the XVII IMEKO World Congress on Metrology in the 3rd Millennium, Dubrovnik, Croatia 2003, pp. 837-840.

${ }^{19}$ M. Fu and G. Chen, IEE Proc. Sci., Meas. Technol., 150, 89 (2003).

${ }^{20}$ H. Kawamura, M. Nawata, and M. Ieda, Electr. Eng. Jpn. 104, 16 (1984).

${ }^{21}$ M. Wissler, "Modeling dielectric elastomer actuators," Ph.D. thesis (Swiss Federal Institute of Technology, 2007).

${ }^{22}$ X. Zhao and Z. Suo, J. Appl. Phys. 104, 123530 (2008).

${ }^{23}$ E. Arruda and M. Boyce, J. Mech. Phys. Solids 41, 389 (1993).

${ }^{24}$ R. Ogden, Proc. R. Soc. London, Ser. A 326, 565 (1972).

${ }^{25}$ O. Yeoh, Rubber Chem. Technol. 66, 754 (1993).

${ }^{26}$ J. Plante and S. Dubowsky, Sens. Actuators, A 137, 96 (2007).

${ }^{27}$ G. Kovacs, L. During, S. Michel, and G. Terrasi, Sens. Actuators, A 155, 299 (2009). 